\title{
Village eLearning: An offline mobile solution to rural communities' knowledge requirement
}

\author{
Souleymane Camara \\ Sujan Shrestha \\ José Abdelnour-Nocera \\ School of Computing - Thames Valley University \\ St Mary's Road - London - UK \\ \{souleymane.camara, sujan.shrestha, jose.abdelnour-nocera, moorejo\}@tvu.ac.uk
}

John Moore

\begin{abstract}
This paper presents an offline mobile eLearning concept as an ICT solution to address the knowledge requirements of a rural sub-Saharan farming community. A socio-technical customisation and deployment of WikiReaders is proposed to support offline mobile access to digital content. A future use scenario is presented as a demonstration of cheap but sustainable innovation emerging from a longitudinal evaluation of users, their needs and their context.
\end{abstract}

Mobile eLearning, socio-technical, sub-Saharan rural communities, ICT for development.

\section{INTRODUCTION}

Mobile access to digital content is improving and is already playing a central role in bridging the digital divide. There are examples of how simple handsets and networks are beginning to produce indispensable services such as M-PESA [10] and HealthNet [13]. We believe that, delivering such services on open hardware and software does not only lower cost in enabling Information and Communication Technologies (ICT) in developing countries, but sustainably increases access to much needed knowledge and learning resources that are readily available and freely accessible.

In this paper, we propose a proof of concept grounded in the requirements and experiences of a recent ICT project. Two rural Kenyan villages in the Village eScience for Life (VeSeL) project were initially introduced to basic ICT under the umbrella of Bridging the Global Digital Divide (BGDD).

The rural communities have now reached a level of adoption to request more tailored and relevant information to improve their farming practices and education in primary schools based on their own contexts and cultures. They also intend to make the most of their newly acquired ICT skills and resources. Based on our experience and sociotechnical assessment of these communities, we propose a customisation of WikiReaders ${ }^{1}$ as a sustainable low cost ICT solution to support their access to knowledge. The paper sets the scene by providing a background of the rural communities' need for access to information as identified by the $\mathrm{VeSeL}^{2}$ project. Next, it explains the rationale for opting for WikiReaders based on the exploration of previous ethnographic and ICT adoption studies conducted by the VeSeL project. The paper also provides the actual socio-technical solution proposed and a plan for its evaluation. Finally, a discussion on how this adaptation could potentially meet the users' requirements is offered prior to a deducted conclusion.

\section{BACKGROUND: VESEL}

Vesel was a research project and part of the bgdd (bridging the global digital divide) initiative ${ }^{3}$. It aimed at identifying and developing suitable information and communication technologies (ict) for groups of rural farmers in kenya, in order to promote e-science in education and provide local communities with access to information to improve their farming practices and the profitability of their produce, thus improving their quality of life. The project lasted for three years from september 2006 to november 2009. Following an initial study in collaboration with a local partner, the University of Nairobi (UoN), two communities in Kenya were identified for a parallel development of ICTs. One site, located in central Kenya in Kiangwaci, focused on the information needs of farmers to plant, tend and bring to market the most appropriate crops for local and EU consumers by making effective use of irrigation and pest control measures. Figure 1 shows the rich Kiangwaci landscape.

The second site in Kambu, in the south of Kenya, has drought problems that needed support via crop selection and irrigation management.

Figure 2 shows the precarious Kambu landscape. In each site, one local primary school and one farming community group were identified.

\footnotetext{
${ }^{1}$ WikiReader: http://thewikireader.com/about.html

${ }^{2}$ VeSeL: http://www.lkl.ac.uk/projects/vesel/

${ }^{3}$ http://www.bgdd.org
} 


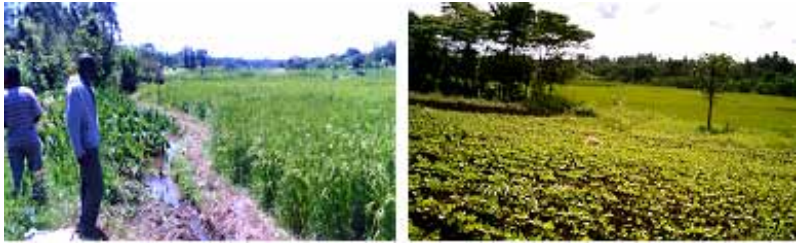

Figure 1: Fields and water in Kiangwaci

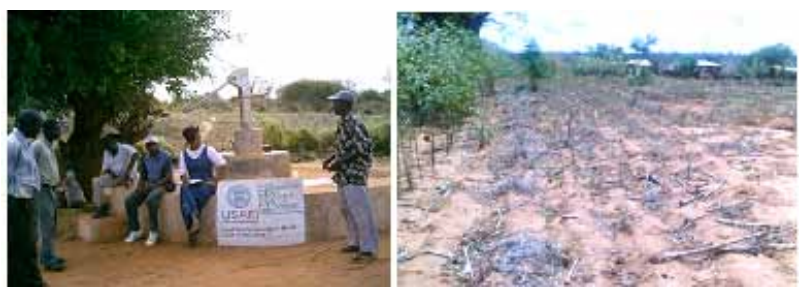

Figure 2: Fields in and Water in Kambu

It is common practice in Kenya for farmers to organise themselves into self-help groups based on the crops they grow, or a buying group for price control and competitive advantage.

Once the communities were identified, VeSeL engaged in identifying cultural and contextual characteristics of the two selected rural communities (Kiangwaci and Kambu) and in identifying fit for purpose solutions. Many studies provide empirical and other technical research on the communities' ethnography and socio-technical context [2, 4, 12]. In early 2008, VeSeL provided a resource kit to farming self-help groups and one primary school in each of the two communities. VeSeL found it crucial to initially provide some minimal communication technologies (a resource kit) to facilitate ongoing interaction and to introduce the targeted rural communities to basic ICTs. The kit they introduced consisted of: an Apple MacBook laptop; an additional desktop; two digital cameras; one MP3 player/recorder, training manuals on each technology; a solar panel charger for the laptop and small devices (phone, camera, etc.).

To date, a very good proportion of those targeted community members have learned to use the kit and other ICT features to a point at which they have been challenging VeSeL to extend the kit to further address their needs.

Further issues identified with the communities involved searching for relevant information based on a current problem they were facing (pests, alternative markets) or immediate need to move on to a new crop (agricultural knowledge on new crops). Primary schools teachers also needed access to teaching and learning resources to improve their practice.

However, because of the limited access to the internet due to cost, availability and bandwidth, users were experiencing difficulties in making the most of ICT for their needs. This hindrance was consequently affecting adoption and use of the existing ICT.

We propose a socio-technical solution using WikiReaders as a low cost open source mobile learning technology to address some of these challenges and issues. Especially, the need for access to knowledge.

\section{RATIONALE FOR A SOCIO-TECHNICAL SOLUTION}

A socio-technical approach to system design is concerned with "the functions of the system" and "the functions of human co-operation"[16]. This approach proposes an equal focus on both the social and technical aspects of the design for a dependable system [7], a system that users can identify with, own and feel part of.

Considering the context and culture of Kiangwaci and Kambu communities, we propose an offline and inter-mediated mobile learning environment using WikiReaders. A WikiReader is an electronic encyclopaedia. It provides the content of Wikipedia on a small memory card (SD card) loaded into a small and portable graphical user interface. See figures 3 and 4 .

The device uses open software allowing developers to customise or adapt it for their purpose.

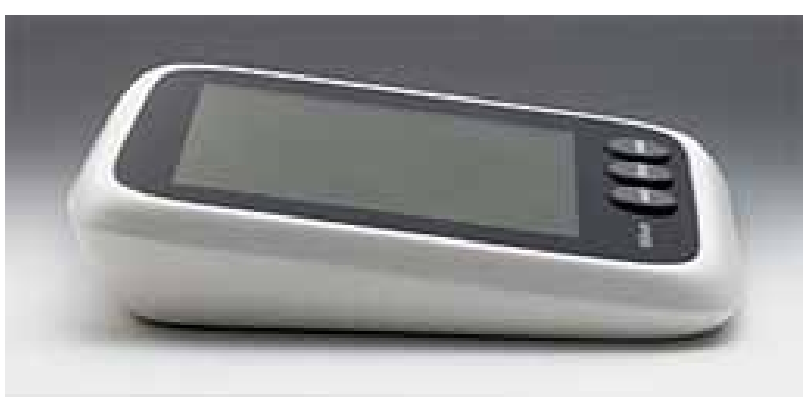

Figure 3: WikiReader profile

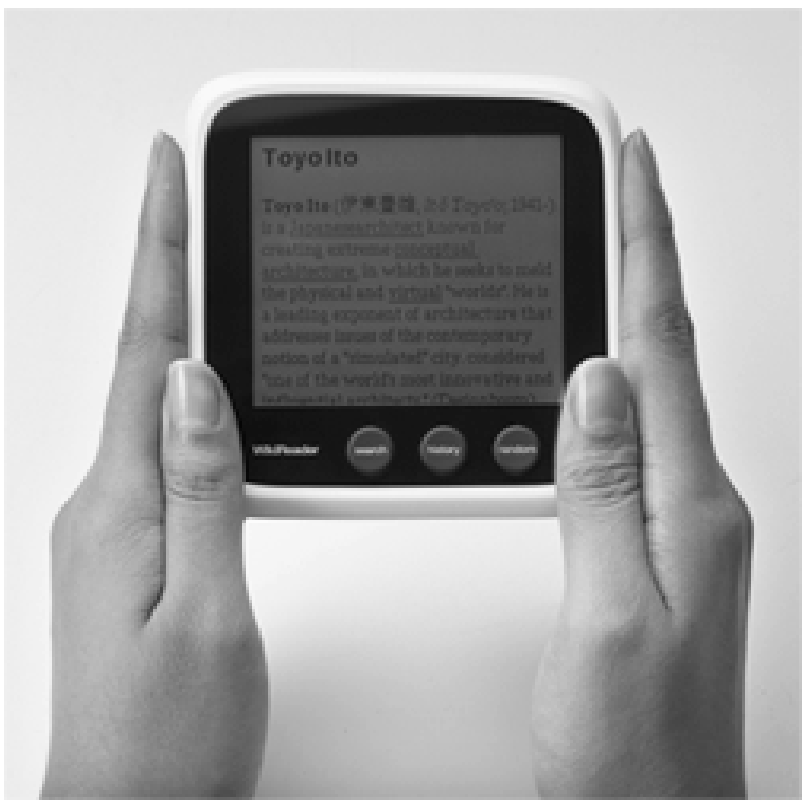

Figure 4: Wiki Reader Interface 
Most importantly, these kinds of devices are not so general purpose as others such as the iPod Touch ${ }^{4}$, thus they are very limited in functionalities.

Because of these limitations, they have minimal resale potential. We see this as positive since they would be more likely to be used for learning purposes rather than being stolen or sold. "One trick pony" devices can be a safe solution.

Furthermore, interaction with a WikiReader is very simple and intuitive with 3 simple buttons and a touch-screen that facilitates typing and scrolling with fingertips. For these rural communities with limited ICT experience, a few interaction points on a device can reduce the time it takes for users to familiarise themselves with the device. But most importantly, this reduces uncertainty.

East Africans are known to have a high uncertainty avoidance index according to Hofstede [8]. This was further explored and validated by another study with these communities [4] which showed that rural Kenyan communities have a high uncertainty avoidance index when using technology. While probing users with the resource kit, users did not do anything they had not been shown before. If there was a malfunction or they did not know how to perform a desired task, they always came back to ask questions. For example, when the screen of the digital camera went to a power saving mode, they never engaged in trying to solve the problem themselves. WikiReaders are limited to searching by keywords (typing through a displayed keyboard), viewing browsing history or randomly exploring content by topics. These limited features are a good fit for purpose given the targeted users' context and culture.

The screen of the WikiReader is designed to facilitate reading under bright sunlight (like in Kambu) as well as indoors or a poorly lit environment. The device also has a scratch resistant tempered glass screen and strong plastic casing for added durability. These rough form-factors would work in the rainy and muddy Kiangwaci village where people share the device.

Providing successful mobile learning services has so far been restricted by the "poor information infrastructure and higher costs of mobile devices and connectivity in developing countries" [17].

A WikiReader runs on two AAA batteries for 12 months in normal usage. Furthermore, the community designing or moulding a device to fit their needs is a participatory customisation [2]. Open hardware projects plus open software projects allow this roadmap. The scenario of use and the sociotechnical approach to this customised offline mobile learning is next discussed.

\subsection{Scenario of Use: Kiangwaci farmers}

We envisaged the following as an example of a deployment and use scenario:

Farmers in Kiangwaci have intermittent need for and access to ICT for farming knowledge. They meet once a week to decide on a common farming issue or pertinent case to explore. At this meeting, farmers write down the issues and illustrate them wherever necessary with pictures using their existing ICT (digital camera and word processor or laptop).

Kiangwaci is only an hour and half's drive to Nairobi. Frequent transport links travel through the village to Nairobi allowing postal services, relatives and government vehicles to come and go.

These transport facilities would help get the aggregated content from Kiangwaci to UoN.

The University of Nairobi (UoN), the local partner, has regular access to the Internet and a great deal of farming knowledge due to an agricultural department with partners in the public and private sectors. At UoN, the agricultural department would search for and prepare relevant content (knowledge) to address farmers' issues. These could be digital instructions or reading materials that would be loaded onto a WikiReader to be sent back to farmers. At UoN a standard Wiki page template would be installed and customised specifically to aggregate content so that it could be easily loaded onto the WikiReaders. The WikiReaders would then be dispatched back to Kiangwaci using the same mode of transport. Upon reception, community members would resume their learning meetings to explore the issues addressed. Individuals could also take the readers to the comfort of their own homes to browse for answers and if necessary, they could reconvene to discuss the knowledge acquired.

Agricultural extension officers provided by the government and the private buyers also expressed the need to use this system to assist their farmers in Kiangwaci to easily access information and knowledge they provided to them. These officers would partner with UoN to supply knowledge resources to be formatted and specified for easy retrieval by the farmers.

The above scenario was discussed to some extent with the different communities but with no mention of the WikiReader.

We merely explored initial feasibility to address their requirements. We also acknowledged that some socio-technical considerations and adaptations would be needed to prove this concept. 


\subsection{Socio-Technical Considerations}

Despite the relatively new technology of WikiReader, the quality has been gradually improved through updates. Scrolling, keyboard and navigation have also been considerably enhanced and tested.

However, an initial question one might ask concerns the literacy and language of the community. According to VeSeL ethnographic research, literacy is quite high in the communities. This is also confirmed by UNICEF5 to be around $76 \%$ in Kenya. Although Kiswahili remains the official language, English is predominant in education and in trade. Each community has enough educated members to facilitate knowledge discovery. The initial resource kit offered by VeSeL has also considerably improved their attitude towards technology or at the very least, provided the community with ICT champions who serve as disseminators and facilitators. We intend to build on these opportunities while we consider any future requirement

Technically, between the UK partners and UoN, which also specialises in computing, we believe we have enough technical know-how to undertake the required customisation. Nonetheless, WikiReader developers also provide to all interested parties some level of support in their customisation.

In terms of sustainability, WikiReaders have a long battery life and currently costs less than US\$ 100. Also, because the device is an open platform, it would be expected that the community of developers would influence its future hardware and software revisions and potentially increase distribution and thus reduce costs. This scenario of use intends to be one proof of the concept and a realistic alternative to the expensive 'One Laptop Per Child' scheme,[6, $15]$.

In both villages, farmers or schools plan one day of the week for either research (Kiangwaci farmers) or preparing and posting one or two best stories on their blog (Silanga School in Kambu).

These stories are usually prepared by a class or a group of students. Given this intermittent knowledge requirements with the exception of the primary schools, it is not necessary to provide one WikiReader per farmer or per child. Rather, a limited number can be assigned for a usage trial.

Nonetheless, for such a concept to be realistic and validated, a great deal of evaluation will need to underpin its design, implementation and use.

\section{PROPOSED EVALUATION OF THE CONCEPT}

The profound implications of culture $[1,8,11]$ and context $[9,14]$ in the design, use and acceptance of information systems (IS) is well documented. But also, in the field of Human Computer Interaction $(\mathrm{HCl})$, there exist validated methods and frameworks to facilitate cultural and contextual elicitation and implication. To effectively and efficiently achieve the proposed solution, we intend to evaluate the processes as a set of socio-technical experiments, [7]. This is an iterative approach that requires evaluation of the different stages of the design process informing the subsequent ones. This will be achieved by the use of sociotechnical evaluation matrices (STEM), [3, 4]. STEM is an interactive collaborative tool that allows every design decision to be evaluated in its implication for both the user and for the technology against pre-defined (agreed) criteria.

Since the design setting will be multi-cultural (UK and Kenya stakeholders) and multi-disciplinary (experts in $\mathrm{HCl}$, computing and agriculture), it is therefore important to make explicit any inherent issues to all stakeholders to ensure no silences or sensibilities are overlooked. We also intend to start the process with a very limited and controlled number of users (teachers, selected farmers). User acceptance and adoption and use of the technology can then be fairly evaluated to inform subsequent development and deployment $[5,18]$.

\section{CONCLUSION}

Designing successful solutions needs an understanding of the contexts and culture of stakeholders on the one hand. On the other, the socio-technical implications of every design and implementation decision also need to be evaluated and made explicit to all stakeholders to verify and validate the system.

The village elearning we have proposed here is rooted in the socio-technical contexts of two rural communities in sub-Saharan Africa (Kiangwaci and Kambu). Experiences from previous interactions with these communities are key to the success of the solution. WikiReaders are proposed as an intermediate offline mobile learning environment to meet the most urgent users' requirements deriving from the VeSeL project. The implementation of WikiReaders in these communities is a clear evidence that innovation to address the BGDD does not need to be expensive.

To ensure success in the proof of this concept, we have proposed a socio-technical approach to designing and evaluating the solution using existing methods, tools and frameworks. It is anticipated that the feasibility of the solution lies in the open platform of WikiReaders and an existing scenario of use.

\section{ACKNOWLEDGMENT}

Our thanks to the VeSeL project for the opportunity and special thank to the Kiangwaci and Kambu communities for their participation. 


\section{REFERENCES}

[1] Abdelnour-Nocera, J., Dunckley, L. and Sharp, $\mathrm{H}$. An Approach to the Evaluation of Usefulness as a Social Construct Using Technological Frames. International Journal of Human-Computer Interaction, 22 (1 \& 2). 153-172.

[2] Camara, S., Abdelnour-Nocera, J., Luckin, R. and Waema, T., Bridging the global digital divide with participatory customisation. in 6th Conference on Cultural Attitudes towards Technology and Communication (CATAC), (Nimes, France, 2008), Ess, C. and Sudweeks, F., (Eds.) 348 - 362.

[3] Camara, S.B., Abdelnour-Nocera, J. and Dunckley, L., Exploring the Problem Domain: A Socio-Technical ICT Design for the Developing World. in 10th Participatory Design Conference, (Bloomington, IN, USA, 2008), PDC08, 154 - 157.

[4] Camara, S.B., Oyugi, C., Abdelnour-Nocera, J. and Smith, A., Augmenting Usability: Cultural elicitation in $\mathrm{HCl}$. in HWID 2009 Conference on Usability in Cultural, Social and Organizational Contexts, (Pune, INDIA, 2009), Springer.

[5] Carlsson, C., Carlsson, J., Hyvonen, K., Puhakainen, J. and Walden, P. Adoption of Mobile Devices/Services: Searching for Answers with the UTAUT Proceedings of the 39th Annual Hawaii International Conference on System Sciences Volume 06, IEEE Computer Society, 2006.

[6] Chhanabhai, P. and Holt, A. One mobile per child: a tractable global health intervention Journal of Health Informatics in Developing Countries, 3 (2). [7] Hansen, R.T., String of Experiments - Looking at the Design Process as a Set of Socio-technical Experiments. in Proceedings of the ninth Participatory Design Conference 2006, (Trento, Italy), ACM.

[8] Hofstede, G. and Hofstede, G.J. Cultures and organizations : software of the mind : [intercultural cooperation and its importance for survival]. McGraw-Hill, New York, 2005.

[9] Honold, P. Culture and Context: An Empirical Study for the Development of a Framework for the Elicitation of Cultural Influence in Product Usage. International Journal of Human-Computer Interaction, 12 (3). 327 - 345.

[10] Hughes, N. and Lonie, S. M-PESA: Mobile Money for the "Unbanked" Turning Cellphones into 24-Hour Tellers in Kenya. Innovations: Technology, Governance, Globalization, 2 (1-2). 63-81.

[11] Jourde, F., Laurillau, Y., Nigay, L. and Moran, A. Conception de systèmes collaboratifs multimodaux: analyse comparative de notations Proceedings of the 20th International Conference of the Association Francophone d'Interaction Homme-Machine, ACM, Metz, France, 2008.

[12] Kevin, W., Joshua, U., Tim, W., Lynne, D., Jos, Abdelnour, N., Rosemary, L., Cecilia, O. and Souleymane, C., A resource kit for participatory socio-technical design in rural kenya. in $\mathrm{CHI}{ }^{\circ} 08$ extended abstracts on Human factors in computing systems, (Florence, Italy, 2008), ACM, 2709-2714.

[13] Mbarika V. W. A. On site: Is telemedicine the panacea for Sub-Saharan Africa's medical nightmare? Communications of the ACM, Vol. 47 (7). $21-24$.

[14] Meso, P., Musa, P. and Mbarika, V. Towards a model of consumer use of mobile information and communication technology in LDCs: the case of sub-Saharan Africa. Information Systems Journal, 15. 119-146.

[15] OLPC. One Laptop per Child, 2008.

[16] Shneiderman, B. and Rose, A. Social impact statements: engaging public participation in information technology design Proceedings of the symposium on Computers and the quality of life, ACM Press 0-89791-827-4/96/02, Philadelpia, Pennsylvania, United States, 1996.

[17] Shrestha, S., Moore, J. and AbdelnourNocera, J. Offline Mobile Learning for ICT4D IADIS International Conference Mobile Learning, Porto, Portugal, 2010.

[18] Viswanath Venkatesh, Michal G Morris, Gordon B Davis and Davis, F.D. User Acceptance of linformation Technology: Toward a Unified View MIS Quaterly, ABI/INFORM Global, 2003, 425-478. 